Ellasia Tan ORCID iD: 0000-0002-8225-9045

Sebastian WoodＯRCID iD: 0000-0002-8574-0475

\title{
A highly sensitive molecular structural probe applied to in-situ biosensing of metabolites using PEDOT:PSS
}

\author{
Ellasia Tan ${ }^{\mathrm{a}}$, Anna-Maria Pappa ${ }^{\mathrm{b}}$, Charalampos Pitsalidis ${ }^{\mathrm{b}}$, James Nightingale ${ }^{\mathrm{a}}$, \\ Sebastian Wood ${ }^{\mathrm{c}}$, Fernando A. Castro ${ }^{\mathrm{c}}$, Roisin M. Owens ${ }^{\mathrm{b}}$ and Ji-Seon Kim ${ }^{\mathrm{a} *}$
}

${ }^{a}$ Department of Physics and Centre for Plastic Electronics, Imperial College London, SW7 2AZ, United Kingdom

${ }^{\mathrm{b}}$ Department of Chemical Engineering and Biotechnology, Philippa Fawcett Drive, CB3 0AS Cambridge, United Kingdom

c National Physical Laboratory, Hampton Road, Teddington, TW11 0LW, United Kingdom

*Correspondence e-mail: ji-seon.kim@imperial.ac.uk

ABSTRACT: A large amount of research within organic biosensors is dominated by organic electrochemical transistors (OECTs) that use conducting polymers such as poly(3,4-ethylene dioxythiophene doped with poly(styrenesulfonate) (PEDOT:PSS). Despite the recent advances in OECT-based biosensors, the sensing is solely reliant on the amperometric detection of the bioanalytes. This is typically accompanied by large undesirable parasitic electrical signals from the electroactive components in the electrolyte. Herein, we present the use of in-situ resonance Raman spectroscopy to probe subtle molecular structural changes of PEDOT:PSS associated with its doping level. We demonstrate how such doping level changes of PEDOT:PSS can be used, for the first time, on operational OECTs for sensitive and selective metabolite sensing whilst simultaneously performing amperometric detection of the analyte. We test the sensitivity by molecularly sensing a lowest glucose concentration of $0.02 \mathrm{mM}$ in phosphate buffered saline (PBS) solution. By changing the electrolyte to cell culture media, the selectivity of in-situ resonance Raman spectroscopy is emphasized as it remains

This article has been accepted for publication and undergone full peer review but has not been through the copyediting, typesetting, pagination and proofreading process, which may lead to differences between this version and the Version of Record. Please cite this article as doi: 10.1002/bit.27187.

This article is protected by copyright. All rights reserved. 
unaffected by other electroactive components in the electrolyte. The application of this molecular structural probe highlights the importance of developing biosensing probes that benefit from high sensitivity of the material's structural and electrical properties whilst being complimentary with the electronic methods of detection.

\section{Graphical Abstract}

A large amount of research within organic biosensors is dominated by organic electrochemical transistors (OECTs) that use conducting polymers such as poly(3,4-ethylene dioxythiophene doped with poly(styrenesulfonate) (PEDOT:PSS).

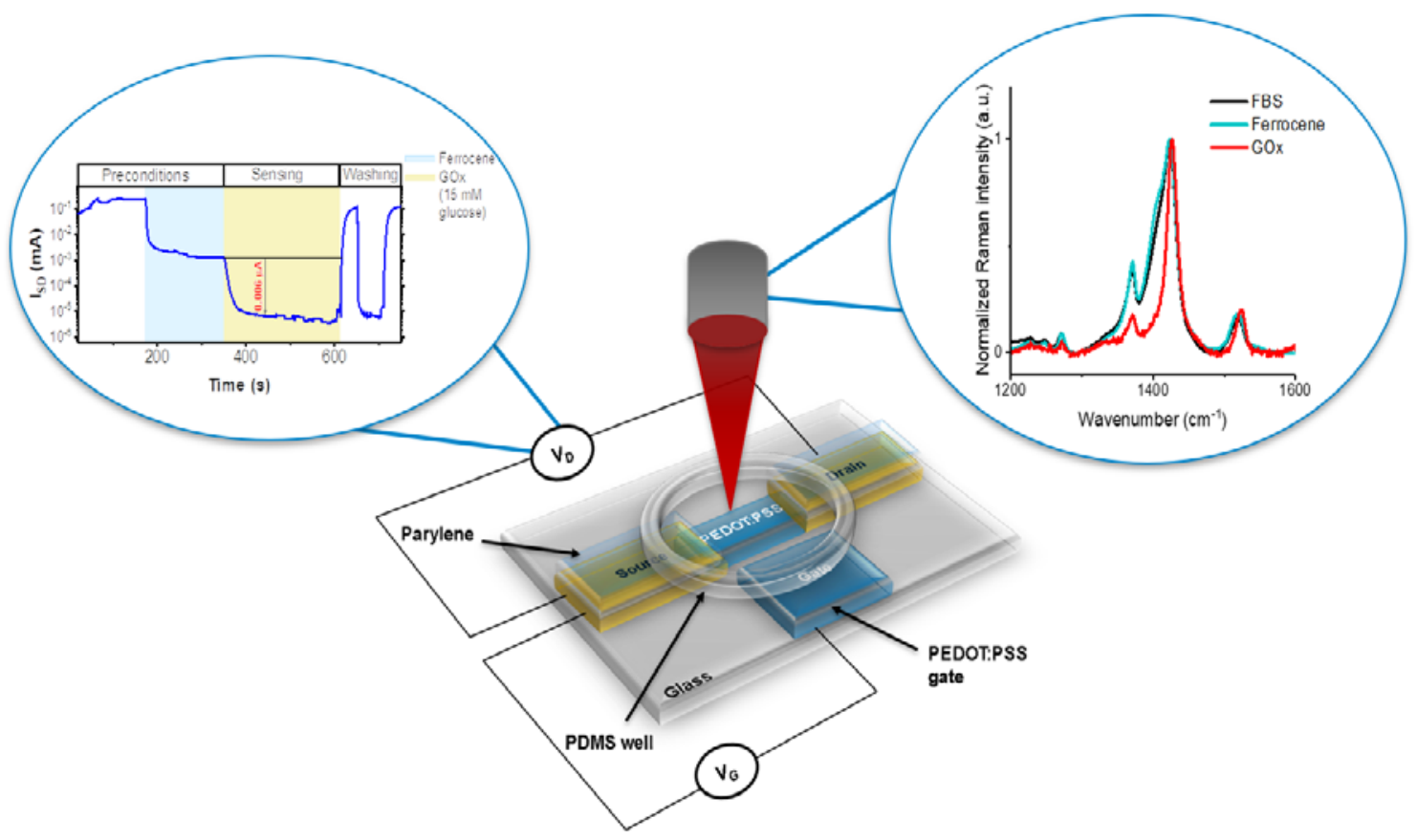

\section{KEYWORDS}

OECT, PEDOT:PSS, metabolite sensing, in-situ, resonance Raman, doping level.

\section{INTRODUCTION}

Conjugated polymers (CP) are widely used for the development of organic electronic devices owing to their low cost, solution processability and mechanical flexibility.(Facchetti, 2011) Poly(3,4-ethylene dioxythiophene doped with poly(styrenesulfonate) (PEDOT:PSS) is one of the most commonly used CPs for bioelectronic applications. This is due to its mixed ionic and electronic conductivity and biocompatibility allowing its direct interfacing with aqueous environments and biological systems whilst preserving its electrical properties.(Strakosas, Wei, Martin, \& Owens, 2016) One notable example of its implementation into novel device configurations for bioelectronics, is the organic electrochemical transistor (OECT).(Rivnay et al., 2018) 
The OECT is a 3-terminal device consisting of source, drain and gate electrodes as well as a mixed conductor channel (mostly the CP PEDOT:PSS) placed between the source and drain electrodes. Typically, an electrolyte is in contact with the channel and the gate electrode, wherein the latter controls the doping level of the CP, via the injection of ions from the electrolyte. This interaction takes place in the whole volume of the CP channel resulting in high efficiency ionic to electronic signal transduction (defined as transconductance).(Rivnay et al., 2013) As such, this inherently sensitive transducer has been used in many biological applications as a platform for transducing and amplifying biological signals (typically ionic) to electronic readouts. A few examples include, sensing of bioanalytes (i.e., metabolites, DNA, etc), neural signals recordings as well as in-vitro toxicology testing.(Curto et al., 2017; A. M. Pappa et al., 2016, 2017; Pitsalidis et al., 2018; Spyropoulos, Gelinas, \& Khodagholy, 2019)

The majority of OECT-based metabolite sensing relies on the amperometric detection of the bioanalyte upon its interaction with a biorecognition element present in the device.(A.-M. Pappa et al., 2018) This technique relies on the drain current output of the device and the sensitivity ranges between $\mathrm{nA}$ - $\mu \mathrm{A}$.(Tang, Yan, Lin, Xu, \& Chan, 2011; Zhang et al., 2015) Although metabolite detection has extended to low mM concentrations, there is usually undesirable electrochemical interference from endogenous electroactive components in the electrolyte (i.e., cell culture media, bio-fluids etc.) thus hindering its practical implementation to some extent. One solution has been to design reference sensors alongside the metabolite sensor to subtract the real baseline(Braendlein et al., 2017) complicating the design of the sensor. Therefore, developing methods that do not rely solely on the electrical readout of the device while still benefiting from the CP properties and its mixed conductivity, can open new directions in molecular sensing in terms of specificity and sensitivity as well as provide straightforward paths toward their real-world implementation.(A.-M. Pappa et al., 2018)

Herein, we demonstrate the use of molecular vibrational Raman spectroscopy as a highly sensitive to the material's chemical structure and electrical properties (i.e., doping level) technique, complimentary to the use of OECTs for enzymatic-based metabolite sensing. Raman spectroscopy is a well-known non-destructive method for probing structural changes, (Wood et al., 2017) such as those induced by biological interactions, thus serving as an excellent molecular sensor. Indeed, it has been successfully integrated into several biosensing applications including cell-based biosensors(Chaichi, 2018), surface-enhanced Raman biosensors(Pilot et al., 2019; Laing, Jamieson, Faulds, \& Graham, 2017) and metabolite biosensors in saliva(Žukovskaja, Jahn, Weber, Cialla-May, \& Popp, 2017). Our work rises above the state of the art by combining an efficient electronic transducer with a structural probe to achieve high sensitivity and gain insight on the molecular interactions

This article is protected by copyright. All rights reserved. 
taking place upon sensing. Although the use of SERS on biological fluids in OECTs has been introduced,(Chou et al., 2019), differing from previous studies, this is the first time that the in-situ resonance Raman spectroscopy has been applied to operational OECTs for metabolite sensing via direct structural changes in the CP (PEDOT:PSS). We first define the vibrational modes and Raman signatures sensitive to PEDOT:PSS doping level and chemical structure. We then demonstrate the use of our structural probe to sense glucose in real-time in an OECT configuration comprised of a PEDOT:PSS channel. Finally, by using cell culture media as the electrolyte, we highlight the specificity and sensitivity of the structural probe due to the absence of parasitic signals, compared to commonly used electronic methods of detection. Further, the simultaneous structural and electrical probing provides a deeper insight on the detection mechanism correlating the structural changes induced on PEDOT:PSS upon enzymatic interactions with the electronic response.

\section{MATERIALS AND METHODS}

\section{Materials}

The PEDOT:PSS formulation used throughout the study was PH1000 (Clevios, Heraeus). For various in-situ electrochemical Raman measurements, the PEDOT:PSS was crosslinked with $1 \% \mathrm{v} / \mathrm{v}$ of (3Glycidyloxypropyl)trimethoxysilane (GOPS). For the electrolyte, $0.1 \mathrm{M} \mathrm{NBu}_{4} \mathrm{PF}_{6}$ in acetonitrile (ACN) and phosphate buffer saline (PBS) solution were used. The additives $0.5 \mathrm{wt} \%$ 4-Dodecylbenzenesulfonic acid (DBSA), $5 \% \mathrm{v} / \mathrm{v}$ ethylene glycol and 1\% v/v GOPS were mixed into the PEDOT:PSS for the device channels. For the electrolyte, PBS solution, ferrocene, glucose oxidase and D-glucose were all bought from Sigma Aldrich and used in the PBS glucose sensing experiments. The complex cell media sensing experiment used Dulbecco's modified eagle medium (DMEM, Sigma Aldrich) containing 10\% fetal bovine serum, $4.5 \mathrm{~g} / \mathrm{L}$ of glucose, L-glutamine, phenol red and other inorganic salts, amino acids and vitamins.

\section{Film and device preparation}

The PEDOT:PSS mixture was sonicated for 10 mins and spin coated onto the substrates. For the electrochemical cell, fluorine-doped tin oxide (FTO) substrates were used after sequential cleaning and sonicating with soap water, acetone and iso-propanol (IPA) for 10 mins each. The FTO substrates were oxygen plasma ashed and the films were prepared by spin-coating PEDOT:PSS/GOPS (1\% v/v) at $1000 \mathrm{rpm}$ for 60s with 3 depositions. The films were then baked after each deposition at $140^{\circ} \mathrm{C}$ for 3 mins.

The OECT devices were oxygen plasma treated prior to spin-coating the PEDOT:PSS mixture at $2000 \mathrm{rpm}$ for 40s. The OECTs were baked at $60^{\circ} \mathrm{C}$ for 60 s and the sacrificial parylene layer was removed. After, the

This article is protected by copyright. All rights reserved. 
devices were further baked at $140^{\circ} \mathrm{C}$ for 1 hour and soaked in de-ionised (DI) water overnight. The polydimethylsiloxane (PDMS) well was made using Sylgard-184 base and curing agent in a 10:1 ratio. The PDMS well contained the electrolyte with a total volume of $200 \mu \mathrm{l}$ containing $20 \mu \mathrm{l}$ of $10 \mathrm{mM}$ ferrocene in ethanol, 500 units/mL glucose oxidase in PBS and D-glucose in PBS to make up the remaining volume to concentrations of $0.02-0.06 \mathrm{mM}$.

\section{Chronoamperometry}

The electrochemical cell comprised of a working electrode (PEDOT:PSS sample), counter electrode (Pt deposited FTO) and a pseudoreference electrode (Ag wire). The working electrode was kept at $0 \mathrm{~V}$ bias with respect to Ag reference for 10s, an oxidation or reduction potential was applied for $40 \mathrm{~s}$ and $0 \mathrm{~V}$ was reapplied for 20s. All electrochemical measurements were taken using the Autolab potentiostat (PGSTAT). Both the insitu Raman and the UV-Vis measurements were taken when the injected current reached steady state during the application of an oxidizing or reducing potential. The spectrophotometer used for the in-situ UV-Vis measurements was a Shimadzu UV-2600 and the substrate contribution to the transmittance was removed using Abs $=\log \left(\% \mathrm{~T}_{\text {substrate }} / \% \mathrm{~T}_{\text {sample }}\right)$.

\section{Resonance Raman spectroscopy}

The resonant Raman measurements were taken using a Renishaw inVia microscope in a back scattering configuration.(Fei et al., 2015; Tsoi et al., 2011) The excitation sources were either a HeNe laser at $633 \mathrm{~nm}$ with $50 \%$ of $12 \mathrm{~mW}$ laser power or at $785 \mathrm{~nm}$ with $50 \%$ of $130 \mathrm{~mW}$ laser power, under 50x magnification. The in-situ resonant Raman spectra were taken using the electrochemical cell and the laser was focused through the back of the working electrode sample. For the OECT devices, the $785 \mathrm{~nm}$ laser was focused through the electrolyte and onto the channel.

\section{Device characterisation and glucose sensing}

The transfer and output curves were recorded using the Keysight B2902A precision source/measure unit. For the OECT glucose sensing experiments, $0.8 \mathrm{~V}\left(\mathrm{~V}_{\mathrm{G}}\right)$ and $-0.3 \mathrm{~V}\left(\mathrm{~V}_{\mathrm{SD}}\right)$ was applied constantly and the sourcedrain current $\left(\mathrm{I}_{\mathrm{SD}}\right)$ response was recorded. The precondition stages in the sensing experiments involved using a $100 \mu$ l electrolyte of PBS, the ferrocene and glucose oxidase solution. During the glucose sensing stages, the Dglucose in PBS solution was added to the electrolyte well to create concentrations of $0.2-0.06 \mathrm{mM}$. For the experiment with cell culture media, the precondition stage used $80 \mu \mathrm{l}$ of DMEM and $20 \mu \mathrm{l}$ of ferrocene solution. $20 \mu \mathrm{l}$ of glucose oxidase solution was added for the sensing regime.

This article is protected by copyright. All rights reserved. 


\section{Air photoemission (APS) and Kelvin probe (KP) measurements}

APS and KP measurements were taken using APS04 (KP Technology) using samples prepared on FTO substrate.(Lee et al., 2018) The substrate was grounded and using UV light excitation (4-7 eV) the HOMO level was established by extrapolating a linear fit of the cube root of photoemission to the set baseline. KP measurements were used to determine Fermi energy levels and this was calculated from the contact potential difference, which was measured by the Kelvin probe vibrating tip. The silver reference determines the work function of the tip prior to the measurement.

\section{RESULTS AND DISCUSSION}

\section{Optical and structural properties of PEDOT:PSS}

In most biosensing applications using OECTs, biochemical interactions induce changes in the doping level of the CP (PEDOT:PSS) thus changing the current that flows in the channel of the device. To understand the effects of electrochemical doping and dedoping on the optical properties of PEDOT:PSS, we first examined its absorption spectra. The electrochemical processes were investigated by applying a range of oxidation and reduction potentials between -1.0 to $1.0 \mathrm{~V}$, as shown in Figure 1a. Note that the PEDOT:PSS films that were investigated were crosslinked with $1 \%$ GOPS v/v. Supplementary Figure S1a-d shows the profile in ACN electrolyte with $0.1 \mathrm{M} \mathrm{NBu}_{4} \mathrm{PF}_{6}$ with a comparison between $1 \%$ GOPS v/v crosslinked and neat PEDOT:PSS films.

In all the spectra, the absorption transition bands are dependent on the doping level of PEDOT:PSS. The absorption spectrum at $0 \mathrm{~V}$ is associated with the majority of PEDOT:PSS being highly doped, as indicated by the appearance of the broad absorption band in the longer wavelengths $(800-1100 \mathrm{~nm})$, originating from the presence of polaron species (doped PEDOT).(Garreau, Louarn, Buisson, Froyer, \& Lefrant, 1999) Upon application of a reduction potential $(-0.2 \mathrm{~V})$, we observe that the polaron band intensity increases and gradually decreases with subsequently higher reducing potentials. The loss of the polaron band is consistent with previous studies, where the bipolaronic absorption band was observed above $1100 \mathrm{~nm}$. (Garreau, Duvail, \& Louarn, 2002) As PEDOT:PSS is originally highly doped, there would be a number of bipolaron species that coexist with polaron and neutral PEDOT segments. A small reduction potential of $-0.2 \mathrm{~V}$ would result in the conversion of the bipolaron to polaron species hence, the initial rise of polaron band intensity. Similarly, the oxidation potentials show that the polaron band increases up to $0.2 \mathrm{~V}$ and further oxidative polarization (doping) causes a gradual decrease in its intensity, due to the formation of bipolaron species that are slightly lessening the amount

This article is protected by copyright. All rights reserved. 
of polaron segments. Upon application of high reduction potentials, the most significant change in absorbance is seen in the shorter wavelengths. The absorbance increase within $500-800 \mathrm{~nm}$ is associated with the $\pi-\pi^{*}$ transition of the neutral PEDOT species.(Lapkowski \& Proń, 2000)

Concurrently, the effect of GOPS used to crosslink PEDOT:PSS films on absorption spectra is investigated. This crosslinking is important in OECT operation as GOPS is commonly used to prevent delamination and dissolution of the PEDOT:PSS film in aqueous electrolytes.(Inal et al., 2014; Rivnay et al., 2015; Stavrinidou et al., 2013) At $-1.0 \mathrm{~V}$, the neat PEDOT:PSS in ACN show a significantly more intense neutral band compared to the crosslinked films (Figure S1g). This indicates a larger amount of dedoped PEDOT and the appearance of the vibronic features in the $-1.0 \mathrm{~V}$ spectrum also suggests that the neat PEDOT:PSS film is further reduced at the same bias. Although there are fewer differences between the neat and crosslinked films at oxidizing potentials (Figure S1h), we suggest that the lesser degree of dedoping of the crosslinked PEDOT:PSS films is due to a lower total population of available sites for neutral PEDOT segments to form.

From the absorption spectra, we determine the laser excitation wavelength required for resonance Raman spectroscopy measurements.(Kim, Ho, Murphy, Baynes, \& Friend, 2002; Limbu et al., 2019; Luke et al., 2019; Soltzberg et al., 2006) At $633 \mathrm{~nm}$, the neutral band can be resonantly excited and this would provide more sensitive structural characterisation to the changes in the dedoped PEDOT. To determine the effect of the crosslinking on the chemical structure of PEDOT:PSS and its energy levels, PEDOT:PSS films crosslinked with GOPS were investigated using resonance Raman spectroscopy, APS and KP (Figure S2). In the $633 \mathrm{~nm}$ Raman spectrum of the pristine PEDOT:PSS film, we observe 4 Raman peaks in the spectrum that are strongly affected by changes in the PEDOT:PSS doping level.(Chia et al., 2007; Garreau et al., 1999) These are the main vibrational modes assigned to the PEDOT:PSS structures in Figure S2. The thiophene $\mathrm{C}=\mathrm{C}$ symmetric and asymmetric vibration correspond to the peaks around $1420 \mathrm{~cm}^{-1}$ and $1530 \mathrm{~cm}^{-1}$ respectively. Whereas, the C-C intra-ring and inter-ring modes are identified to be around $1370 \mathrm{~cm}^{-1}$ and $1270 \mathrm{~cm}^{-1}$ respectively. Only PEDOT vibrational modes are visible in Raman spectra as PSS does not possess $\pi$ electron delocalisation along the conjugated backbone thus exhibiting significantly lower Raman scattering. In Figure S2a, we observe a $\mathrm{C}=\mathrm{C}_{\text {sym }}$ peak FWHM narrowing and reduced relative intensity of $\mathrm{C}=\mathrm{C}_{\text {asym }}$ to $\mathrm{C}=\mathrm{C}_{\text {sym }}$ with increased concentrations of GOPS (0, 0.5 and $1 \% \mathrm{v} / \mathrm{v})$. Such changes can be interpreted as a structural transformation towards the benzoid conformation represented schematically in Figure S2 (neutral PEDOT). This indicates a greater fraction of dedoped PEDOT as a result of GOPS crosslinking. Concomitantly, in Figure S2b, the Fermi energy level shifts from 4.95 to $4.76 \mathrm{eV}$ (neat and 1\% v/v PP:GOPS respectively) while maintaining the highest occupied

This article is protected by copyright. All rights reserved. 
molecular orbital (HOMO) values of PEDOT:PSS show a negligible change from 4.99 to $5.01 \mathrm{eV}$. The energy gap between the HOMO and Fermi energy level indicates the degree of doping in PEDOT:PSS. Only 0.5\% v/v of GOPS was enough to dedope PEDOT:PSS and change the Fermi energy level from 4.95 to $4.82 \mathrm{eV}$. We note that it has been previously argued that the PEDOT:PSS crosslinking by GOPS does not change its doping level and only affects morphology.(Håkansson et al., 2017) However, the distinct changes in Raman signatures and energetics measured here suggest there is an undesirable dedoping process that occurs upon GOPS crosslinking.

Similarly, PEDOT:PSS doping level is controlled and correlated to its chemical structural changes by using in-situ electrochemical Raman spectroscopy. Excitation wavelengths of 633 and $785 \mathrm{~nm}$ were used to be in resonance with the neutral and polaronic transitions respectively. Figure 1b shows the background subtracted and normalized Raman spectra (inset) using $785 \mathrm{~nm}$ in the reduction potentials. The full Raman spectra corresponding to all reduction and oxidation potentials at both 633 and $785 \mathrm{~nm}$ are shown in Figure S3. In the raw Raman spectra, we can identify the dependence of the Raman intensity on the direction of the applied bias and absorbance. The overall trends for the Raman spectral changes and absorbance is summarised in Figure 1c. We observe the increase of the maximum $\mathrm{C}=\mathrm{C}_{\text {sym }}$ Raman intensity particularly at $633 \mathrm{~nm}$ excitation, as reduction potentials from -0.2 to $-1.0 \mathrm{~V}$ are applied. This due to an increasing amount of the neutral polymer, which is also observed by the absorbance spectra as the neutral band rises. Since the dedoped PEDOT:PSS is the most Raman active at $633 \mathrm{~nm}$, the Raman scattering intensity is positively correlated to the absorbance in the reduction potentials.

At both 633 and $785 \mathrm{~nm}$, there are distinct signatures associated with the doping level change in PEDOT:PSS that can be investigated by using the normalized Raman spectra. The most noteworthy features that can be extracted are the changes in the $\mathrm{C}=\mathrm{C}_{\text {sym }}$ maximum peak position and full width half maximum (FWHM). The shift in $\mathrm{C}=\mathrm{C}_{\text {sym }}$ maximum peak position is pronounced in the oxidation regime, where there is a gradual shift to higher vibrational energy that can be understood as shortening of the effective conjugation length.(Sakamoto, Okumura, Zhao, \& Furukawa, 2005) This also correlates to the absorbance data, which showed that the neutral and polaron band were not fully quenched at $1.0 \mathrm{~V}$ and a bipolaron transition band is expected. This suggests the presence of some neutral, polaron and bipolaron species during the oxidation bias. The subtle difference in peak position for the reduction potentials is caused by the change in the relative intensities of high and low vibrational energy contributions to this peak. This also inadvertently narrows the FWHM of the peak. PEDOT:PSS possesses an intrinsically disordered nature with an extensive amount of polymer conformations.(Dimitriev, Piryatinski, \& Pud, 2011; Rivnay et al., 2016) As such, the narrowing of the $\mathrm{C}=\mathrm{C}_{\text {sym }}$

This article is protected by copyright. All rights reserved. 
FWHM indicates a smaller distribution of the PEDOT conformations when dedoped. We observe the opposite effect when oxidising the film as there is FWHM broadening induced by the higher degree of conformational disorder as a mixture of charged states is present. The Raman signatures that can be used to determine the doping level of PEDOT:PSS are not limited to the $\mathrm{C}=\mathrm{C}_{\text {sym }}$ peak changes. The final panel of Figure $1 \mathrm{c}$ shows that the relative peak intensity of $\mathrm{C}-\mathrm{C}_{\mathrm{inter}}$ to $\mathrm{C}=\mathrm{C}_{\mathrm{sym}}$ is another signature unique to changes in PEDOT:PSS doping level. Above $0.35 \mathrm{~V}$, the PEDOT:PSS may adopt another structural change where the bipolaron species has slightly decreased $\pi$ electron density in the C- $C_{\text {inter }}$ bond.(Garreau et al., 1999) This also correlates with the absorbance, where we see lower polaron band intensity with escalating oxidation bias. Using this electrochemical method, we dedope and dope PEDOT:PSS thus systematically modifying the charge density in the film (Figure S4). We are then able to establish the signatures in absorbance and resonance Raman spectroscopy during the electrochemical doping and dedoping process for PEDOT:PSS.

\section{Biosensing}

We now use the resonance Raman to probe the PEDOT:PSS active channel in an OECT for in-situ measurements. The structural characterisation of the electrochemical doping and dedoping of PEDOT:PSS is relevant to OECT operation and in biosensing as most biological events cause changes in the gating efficiency which ultimate leads to more dedoping of PEDOT:PSS in the channel. The use of the $785 \mathrm{~nm}$ excitation wavelength, which is resonant with the polaron species, ensures that the structural probe does not saturate during the reduction potentials. The OECT device characteristics including output, transfer and transconductance curves, are all shown in Figure S5. We demonstrate the use of the structural probe on a glucose OECT sensor that functions via a ferrocene-mediated mechanism.(Shim et al., 2009) Note that all the components involved in the electron transfer reactions shown in Figure 2 including glucose, glucose oxidase (GOx) and ferrocene (Fc) are present in the electrolyte. Generally, upon interaction of glucose with GOx, the enzyme gets reduced and transfers electrons to the artificial electron acceptor, $\mathrm{Fc}^{+}$, present in the solution. In turn, the reacted mediator transfers charge to the sensing electrode (i.e., PEDOT:PSS) which can be probed electrochemically. For instance, this reaction changes the electrolyte potential thus increasing the gating efficiency, further dedoping the PEDOT:PSS film at the same gate bias. Therefore, a decrease in the current upon the addition of glucose is expected. As such, we use subsequent additions of glucose starting from 0.02 to $0.06 \mathrm{mM}$ to observe any further changes in the current. Figure 2a shows the drain current output $\left(\mathrm{I}_{\mathrm{SD}}\right)$ over time. It is important to note that the drain current is already significantly influenced by the addition of the enzyme and mediator as the $\mathrm{I}_{\mathrm{SD}}$ decreases from 0.6 to $0.15 \mathrm{~mA}$. With the first addition of $0.02 \mathrm{mM}$ glucose the $\mathrm{I}_{\mathrm{SD}}$

This article is protected by copyright. All rights reserved. 
drops to $0.38 \mu \mathrm{A}$, as PEDOT:PSS is further dedoped from the increase in gating efficiency due to the glucose and GOx interaction. When the electrolyte is washed using PBS solution, the current recovers demonstrating the reversibility of the OECT.

Concurrently, the structural probe was used to monitor the Raman spectral changes at each stage in Figure 2b. The top spectra show the preconditioning stage, and this is accompanied by insignificant changes in the peaks. During glucose additions, the spectra show the PEDOT:PSS dedoping characteristics which were identified previously. These trends are summarised in Figure 2c, where the first panel describes the $\mathrm{C}=\mathrm{C}_{\text {sym }}$ FWHM shift as the peak becomes narrow once $0.02 \mathrm{mM}$ glucose is introduced. As seen in the relative peak areas of the $\mathrm{C}=\mathrm{C}_{\text {sym }}$ mode, the dedoping of PEDOT:PSS affects the lower energy modes first. Such changes correspond to the formation of the benzoid structure with more double bond character, which is higher in energy.(Chiu, Travaš-Sejdić, Cooney, \& Bowmaker, 2005; Łapkowski \& Proń, 2000) The relative integrated peak areas also show that the signature is actually opposite in the pre-conditions stage where there is a slight increase in the lower energy $\mathrm{C}=\mathrm{C}$ mode. In comparison to the amperometric detection, the highlighted blue region of the Raman signatures show that the structural probe is not sensitive to the pre-conditioning (enzyme and mediator). In this experiment, the lowest glucose concentration detected was $0.02 \mathrm{mM}$ in PBS but to be physiologically relevant and simulate real-world metabolite sensing conditions, cell culture media was used to highlight the selectivity of our structural probe.

For this experiment, we test the OECT device using a DMEM solution that contains fetal bovine serum with a biologically relevant glucose concentration $(15 \mathrm{mM})$. Cell culture medium contains several electroactive components, which can influence the drain current output, at the given potential(Yao \& Asayama, 2017) as seen in the precondition stage of Figure 3a. The magnitude of the current drastically drops to the $\mu \mathrm{A}$ range when $\mathrm{Fc}$ is added. Consequently, the glucose sensing is now limited to $\Delta \mathrm{I}_{\mathrm{SD}}$ of $1.2 \mu \mathrm{A}$, as the $\mathrm{I}_{\mathrm{SD}}$ drops to $0.006 \mu \mathrm{A}$. In contrast to the electrical characteristics, the $\mathrm{C}=\mathrm{C}_{\text {sym }}$ FWHM increases as ferrocene is added (Figure $3 b$ ), differing from the dedoping signatures. This suggests that the drain current decrease is not due to the formation of neutral PEDOT. Such changes in the current could be due to the redistribution of ions in the electrolyte as ferrocene is added, which would affect the ionic current in the PEDOT:PSS film. This is undesirable and limits the sensitivity of the electrical probe for the biosensing. When GOx is added to complete the electron transfer chain, the FWHM is reduced by a total of $24 \mathrm{~cm}^{-1}$ to resemble the dedoped PEDOT:PSS. Similarly, the relative peak area of the $\mathrm{C}=\mathrm{C}_{\text {sym }}$ lower to higher energy mode is plotted and the trend shows a decrease that plateaus within 100s (Figure 3c). The normalized spectra for the transient measurements can be seen in Figure S6. Most

This article is protected by copyright. All rights reserved. 
importantly, these results confirm that the resonant Raman spectroscopy is a highly selective transducer for the reduction and oxidation of PEDOT:PSS. The Raman signatures were affected only during sensing thus selectively responding to the glucose biological event, not influenced by other components in the electrolyte. The structural probe exhibited good sensitivity of glucose detection for physiologically relevant applications (glucose levels within blood samples range between 4 - 22 mM). (Li et al., 2013; Razzak et al., 2018) Taking into consideration the prospect of miniaturized Raman spectrometers,(Gnyba, Smulko, Kwiatkowski, \& Wierzba, 2011; Jiang et al., 2013) the structural probe could be easier implemented into real-world biosensing applications.

\section{CONCLUSIONS}

We have investigated the optical properties of PEDOT:PSS, which was followed by tuning the doping level through GOPS crosslinking and electrochemical methods. The structural probe and energetics revealed that the GOPS crosslinker dedoped PEDOT:PSS in exchange for mechanical stability. The in-situ electrochemical Raman was beneficial in gaining an understanding of the spectral features related to the dedoped and doped PEDOT structures. This defined the Raman signatures such as peak position, FWHM and relative peak intensities associated with the degree of doping of PEDOT. The main spectral features related to dedoped PEDOT:PSS are the narrowing of C=Csym FWHM leading to a slight shift in peak position to higher energy and other peaks' relative intensities to C=Csym. This was followed by the combination of the structural probe with the electrical measurements in the biosensing OECT devices. We demonstrated that glucose can be detected through Raman signatures with good sensitivity for physiologically relevant applications. Based on our observations, the drain current fluctuates and responds to the components in the cell culture media particularly in the preconditioning stages. However, the Raman signatures are not affected by these electroactive components highlighting the selectivity of the structural probe. In this work, we have shown that resonance Raman spectroscopy can be used as a non-destructive selective structural probe for enzymatic biosensing. As well as this, we showed that the structural and electrical changes can be simultaneously measured as a result of using operational OECTs. Therefore, we believe that Raman spectroscopy is a powerful and complimentary method that can be easily integrated into biosensing applications.

This article is protected by copyright. All rights reserved. 


\section{ACKNOWLEDGEMENTS}

The authors acknowledge funding from the UK EPSRC for the Plastic Electronics Centre for Doctoral Training (EP/L016702/1) and from the UK Department for Business, Energy and Industrial Strategy (BEIS) through the National Measurement System. The authors thank Prof. James Durrant and Dr. Andreas Kafizas of Imperial College London for the use of the spectroelectrochemistry equipment.

\section{CONFLICTS OF INTEREST}

The authors declare no financial or commercial conflict of interest.

\section{REFERENCES}

Braendlein, M., Pappa, A.-M., Ferro, M., Lopresti, A., Acquaviva, C., Mamessier, E., ... Owens, R. M. (2017). Lactate Detection in Tumor Cell Cultures Using Organic Transistor Circuits. Advanced Materials, 29(13), 1605744. https://doi.org/10.1002/adma.201605744

Chaichi, A., Prasad, A., \& Gartia, M. (2018). Raman Spectroscopy and Microscopy Applications in Cardiovascular Diseases: From Molecules to Organs. Biosensors, 8(4), 107. https://doi.org/10.3390/bios8040107

Chia, P.-J., Chua, L.-L., Sivaramakrishnan, S., Zhuo, J.-M., Zhao, L.-H., Sim, W.-S., ... Ho, P. K.-H. (2007). Injectioninduced De-doping in a Conducting Polymer during Device Operation: Asymmetry in the Hole Injection and Extraction Rates. Advanced Materials, 19(23), 4202-4207. https://doi.org/10.1002/adma.200700086

Chiu, W. W., Travaš-Sejdić, J., Cooney, R. P., \& Bowmaker, G. A. (2005). Spectroscopic and conductivity studies of doping in chemically synthesized poly(3,4-ethylenedioxythiophene). Synthetic Metals, 155(1), 80-88. https://doi.org/10.1016/j.synthmet.2005.06.012

Chou, J.-A., Chung, C.-L., Ho, P.-C., Luo, C.-H., Tsai, Y.-H., Wu, C.-K., ... Chen, P. (2019). Organic Electrochemical Transistors/SERS-Active Hybrid Biosensors Featuring Gold Nanoparticles Immobilized on Thiol-Functionalized PEDOT Films. Frontiers in Chemistry, 7. https://doi.org/10.3389/fchem.2019.00281

Curto, V. F., Marchiori, B., Hama, A., Pappa, A.-M., Ferro, M. P., Braendlein, M., ... Owens, R. M. (2017). Organic transistor platform with integrated microfluidics for in-line multi-parametric in vitro cell monitoring. Microsystems \& Nanoengineering, 3(March), 17028. https://doi.org/10.1038/micronano.2017.28

Dimitriev, O. P., Piryatinski, Y. P., \& Pud, A. A. (2011). Evidence of the controlled interaction between PEDOT and PSS in the PEDOT:PSS complex via concentration changes of the complex solution. Journal of Physical Chemistry B, 115(6), 1357-1362. https://doi.org/10.1021/jp110545t

Facchetti, A. (2011). $\pi$-Conjugated Polymers for Organic Electronics and Photovoltaic Cell Applications †. Chemistry of Materials, 23(3), 733-758. https://doi.org/10.1021/cm102419z

Fei, Z., Boufflet, P., Wood, S., Wade, J., Moriarty, J., Gann, E., ... Heeney, M. (2015). Influence of Backbone Fluorination in Regioregular Poly(3-alkyl-4-fluoro)thiophenes. Journal of the American Chemical Society, 137(21), 6866-6879. https://doi.org/10.1021/jacs.5b02785

Garreau, S., Duvail, J. L., \& Louarn, G. (2002). Spectroelectrochemical studies of poly(3,4-ethylenedioxythiophene) in aqueous medium. Synthetic Metals, 125(3), 325-329. https://doi.org/10.1016/S0379-6779(01)00397-6

Garreau, S., Louarn, G., Buisson, J. P., Froyer, G., \& Lefrant, S. (1999). In Situ Spectroelectrochemical Raman Studies of Poly(3,4-ethylenedioxythiophene) (PEDT). Macromolecules, 32(20), 6807-6812. https://doi.org/10.1021/ma9905674

Gnyba, M., Smulko, J., Kwiatkowski, A., \& Wierzba, P. (2011). Portable Raman spectrometer - design rules and applications. Bulletin of the Polish Academy of Sciences: Technical Sciences, 59(3), 325-329.

This article is protected by copyright. All rights reserved. 
https://doi.org/10.2478/v10175-011-0040-z

Håkansson, A., Han, S., Wang, S., Lu, J., Braun, S., Fahlman, M., ... Fabiano, S. (2017). Effect of (3glycidyloxypropyl)trimethoxysilane (GOPS) on the electrical properties of PEDOT:PSS films. Journal of Polymer Science Part B: Polymer Physics, 55(10), 814-820. https://doi.org/10.1002/polb.24331

Inal, S., Rivnay, J., Leleux, P., Ferro, M., Ramuz, M., Brendel, J. C., ... Malliaras, G. G. (2014). A High Transconductance Accumulation Mode Electrochemical Transistor. Advanced Materials, 26(44), 7450-7455. https://doi.org/10.1002/adma.201403150

Jiang, X., Yang, M., Meng, Y., Jiang, W., \& Zhan, J. (2013). Cysteamine-Modified Silver Nanoparticle Aggregates for Quantitative SERS Sensing of Pentachlorophenol with a Portable Raman Spectrometer. ACS Applied Materials \& Interfaces, 5(15), 6902-6908. https://doi.org/10.1021/am401718p

Kim, J.-S., Ho, P. K. H., Murphy, C. E., Baynes, N., \& Friend, R. H. (2002). Nature of Non-emissive Black Spots in Polymer Light-Emitting Diodes by In-Situ Micro-Raman Spectroscopy. Advanced Materials, 14(3), 206-209. https://doi.org/10.1002/1521-4095(20020205)14:3<206::AID-ADMA206>3.0.CO;2-J

Laing, S., Jamieson, L. E., Faulds, K., \& Graham, D. (2017). Surface-enhanced Raman spectroscopy for in vivo biosensing. Nature Reviews Chemistry, 1(8), 0060. https://doi.org/10.1038/s41570-017-0060

Łapkowski, M., \& Proń, A. (2000). Electrochemical oxidation of poly(3,4-ethylenedioxythiophene) — "in situ” conductivity and spectroscopic investigations. Synthetic Metals, 110(1), 79-83. https://doi.org/10.1016/S03796779(99)00271-4

Lee, S., Kim, D. Bin, Hamilton, I., Daboczi, M., Nam, Y. S., Lee, B. R., ... Song, M. H. (2018). Control of Interface Defects for Efficient and Stable Quasi-2D Perovskite Light-Emitting Diodes Using Nickel Oxide Hole Injection Layer. Advanced Science, 5(11), 1801350. https://doi.org/10.1002/advs.201801350

Li, G., Cabanero, M., Wang, Z., Wang, H., Huang, T., Alexis, H., ... Pincus, M. R. (2013). Comparison of glucose determinations on blood samples collected in three types of tubes. Annals of Clinical and Laboratory Science, 43(3), 278-284.

Limbu, S., Pont, S., Doust, A. B., Kwon, S., Fuller, P., Tan, E., ... Kim, J.-S. (2019). Impact of Initial Bulk-Heterojunction Morphology on Operational Stability of Polymer:Fullerene Photovoltaic Cells. Advanced Materials Interfaces, 6(6), 1801763. https://doi.org/10.1002/admi.201801763

Luke, J., Speller, E. M., Wadsworth, A., Wyatt, M. F., Dimitrov, S., Lee, H. K. H., ... Kim, J.-S. (2019). Twist and Degrade-Impact of Molecular Structure on the Photostability of Nonfullerene Acceptors and Their Photovoltaic Blends. Advanced Energy Materials, 1803755, 1803755. https://doi.org/10.1002/aenm.201803755

Pappa, A.-M., Parlak, O., Scheiblin, G., Mailley, P., Salleo, A., \& Owens, R. M. (2018). Organic Electronics for Point-ofCare Metabolite Monitoring. Trends in Biotechnology, 36(1), 45-59. https://doi.org/10.1016/j.tibtech.2017.10.022

Pappa, A. M., Curto, V. F., Braendlein, M., Strakosas, X., Donahue, M. J., Fiocchi, M., ... Owens, R. M. (2016). Organic Transistor Arrays Integrated with Finger-Powered Microfluidics for Multianalyte Saliva Testing. Advanced Healthcare Materials, 5(17), 2295-2302. https://doi.org/10.1002/adhm.201600494

Pappa, A. M., Inal, S., Roy, K., Zhang, Y., Pitsalidis, C., Hama, A., ... Owens, R. M. (2017). Polyelectrolyte Layer-byLayer Assembly on Organic Electrochemical Transistors. ACS Applied Materials and Interfaces, 9(12), 1042710434. https://doi.org/10.1021/acsami.6b15522

Pilot, Signorini, Durante, Orian, Bhamidipati, \& Fabris. (2019). A Review on Surface-Enhanced Raman Scattering. Biosensors, 9(2), 57. https://doi.org/10.3390/bios9020057

Pitsalidis, C., Ferro, M. P., Iandolo, D., Tzounis, L., Inal, S., \& Owens, R. M. (2018). Transistor in a tube: A route to threedimensional bioelectronics. Science Advances, 4(10), eaat4253. https://doi.org/10.1126/sciadv.aat4253

Razzak, R. A., Alshaiji, A. F., Qareeballa, A. A., Mohamed, M. W., Bagust, J., \& Docherty, S. (2018). High-normal blood glucose levels may be associated with decreased spatial perception in young healthy adults. PLOS ONE, 13(6), e0199051. https://doi.org/10.1371/journal.pone.0199051

Rivnay, J., Inal, S., Collins, B. A., Sessolo, M., Stavrinidou, E., Strakosas, X., ... Malliaras, G. G. (2016). Structural control of mixed ionic and electronic transport in conducting polymers. Nature Communications, 7, 11287. https://doi.org/10.1038/ncomms11287

This article is protected by copyright. All rights reserved. 
Rivnay, J., Inal, S., Salleo, A., Owens, R. M., Berggren, M., \& Malliaras, G. G. (2018). Organic electrochemical transistors. Nature Reviews Materials, 3(2), 17086. https://doi.org/10.1038/natrevmats.2017.86

Rivnay, J., Leleux, P., Ferro, M., Sessolo, M., Williamson, A., Koutsouras, D. a., ... Malliaras, G. G. (2015). Highperformance transistors for bioelectronics through tuning of channel thickness. Science Advances, 1(4), e1400251. https://doi.org/10.1126/sciadv.1400251

Rivnay, J., Leleux, P., Sessolo, M., Khodagholy, D., Hervé, T., Fiocchi, M., \& Malliaras, G. G. (2013). Organic electrochemical transistors with maximum transconductance at zero gate bias. Advanced Materials, 25(48), 70107014. https://doi.org/10.1002/adma.201303080

Sakamoto, S., Okumura, M., Zhao, Z., \& Furukawa, Y. (2005). Raman spectral changes of PEDOT-PSS in polymer lightemitting diodes upon operation. Chemical Physics Letters, 412(4-6), 395-398. https://doi.org/10.1016/j.cplett.2005.07.040

Shim, N. Y., Bernards, D., Macaya, D., DeFranco, J., Nikolou, M., Owens, R., \& Malliaras, G. (2009). All-Plastic Electrochemical Transistor for Glucose Sensing Using a Ferrocene Mediator. Sensors, 9(12), 9896-9902. https://doi.org/10.3390/s91209896

Soltzberg, L. J., Slinker, J. D., Flores-Torres, S., Bernards, D. A., Malliaras, G. G., Abruña, H. D., ... Goldberg, V. (2006). Identification of a Quenching Species in Ruthenium Tris-Bipyridine Electroluminescent Devices. Journal of the American Chemical Society, 128(24), 7761-7764. https://doi.org/10.1021/ja055782g

Spyropoulos, G. D., Gelinas, J. N., \& Khodagholy, D. (2019). Internal ion-gated organic electrochemical transistor: A building block for integrated bioelectronics. Science Advances, 5(2), eaau7378. https://doi.org/10.1126/sciadv.aau7378

Stavrinidou, E., Leleux, P., Rajaona, H., Khodagholy, D., Rivnay, J., Lindau, M., ... Malliaras, G. G. (2013). Direct measurement of ion mobility in a conducting polymer. Advanced Materials, 25(32), 4488-4493. https://doi.org/10.1002/adma.201301240

Strakosas, X., Wei, B., Martin, D. C., \& Owens, R. M. (2016). Biofunctionalization of polydioxythiophene derivatives for biomedical applications. Journal of Materials Chemistry B, 4(29), 4952-4968. https://doi.org/10.1039/C6TB00852F

Tang, H., Yan, F., Lin, P., Xu, J., \& Chan, H. L. W. (2011). Highly Sensitive Glucose Biosensors Based on Organic Electrochemical Transistors Using Platinum Gate Electrodes Modified with Enzyme and Nanomaterials. Advanced Functional Materials, 21(12), 2264-2272. https://doi.org/10.1002/adfm.201002117

Tsoi, W. C., James, D. T., Kim, J. S., Nicholson, P. G., Murphy, C. E., Bradley, D. D. C., ... Kim, J.-S. (2011). The Nature of In-Plane Skeleton Raman Modes of P3HT and Their Correlation to the Degree of Molecular Order in P3HT:PCBM Blend Thin Films. Journal of the American Chemical Society, 133(25), 9834-9843. https://doi.org/10.1021/ja2013104

Wood, S., Hollis, J. R., \& Kim, J.-S. (2017). Raman spectroscopy as an advanced structural nanoprobe for conjugated molecular semiconductors. Journal of Physics D: Applied Physics, 50(7), 073001. https://doi.org/10.1088/1361$6463 / 50 / 7 / 073001$

Yao, T., \& Asayama, Y. (2017). Animal-cell culture media: History, characteristics, and current issues. Reproductive Medicine and Biology, 16(2), 99-117. https://doi.org/10.1002/rmb2.12024

Zhang, M., Liao, C., Mak, C. H., You, P., Mak, C. L., \& Yan, F. (2015). Highly sensitive glucose sensors based on enzymemodified whole-graphene solution-gated transistors. Scientific Reports, 5(1), 8311. https://doi.org/10.1038/srep08311

Žukovskaja, O., Jahn, I., Weber, K., Cialla-May, D., \& Popp, J. (2017). Detection of Pseudomonas aeruginosa Metabolite Pyocyanin in Water and Saliva by Employing the SERS Technique. Sensors, 17(8), 1704. https://doi.org/10.3390/s17081704 


\section{Figure legend}

Figure 1. a) Spectroelectrochemistry absorption spectra of GOPS blended PEDOT:PSS films immersed in PBS electrolyte under applied reduction potentials ( 0 to $-1.0 \mathrm{~V})$. All absorbance bands originate from PEDOT:PSS as GOPS gives no signal in absorbance. b) The in-situ electrochemical Raman spectra with inset normalised spectra of the PEDOT:PSS/GOPS blended film ( $1 \% \mathrm{v} / \mathrm{v})$ under reduction potentials( 0 to $-1.0 \mathrm{~V})$. c) A summary of the absorbance and Raman peak trends at 633 and 785 nm excitation wavelengths.
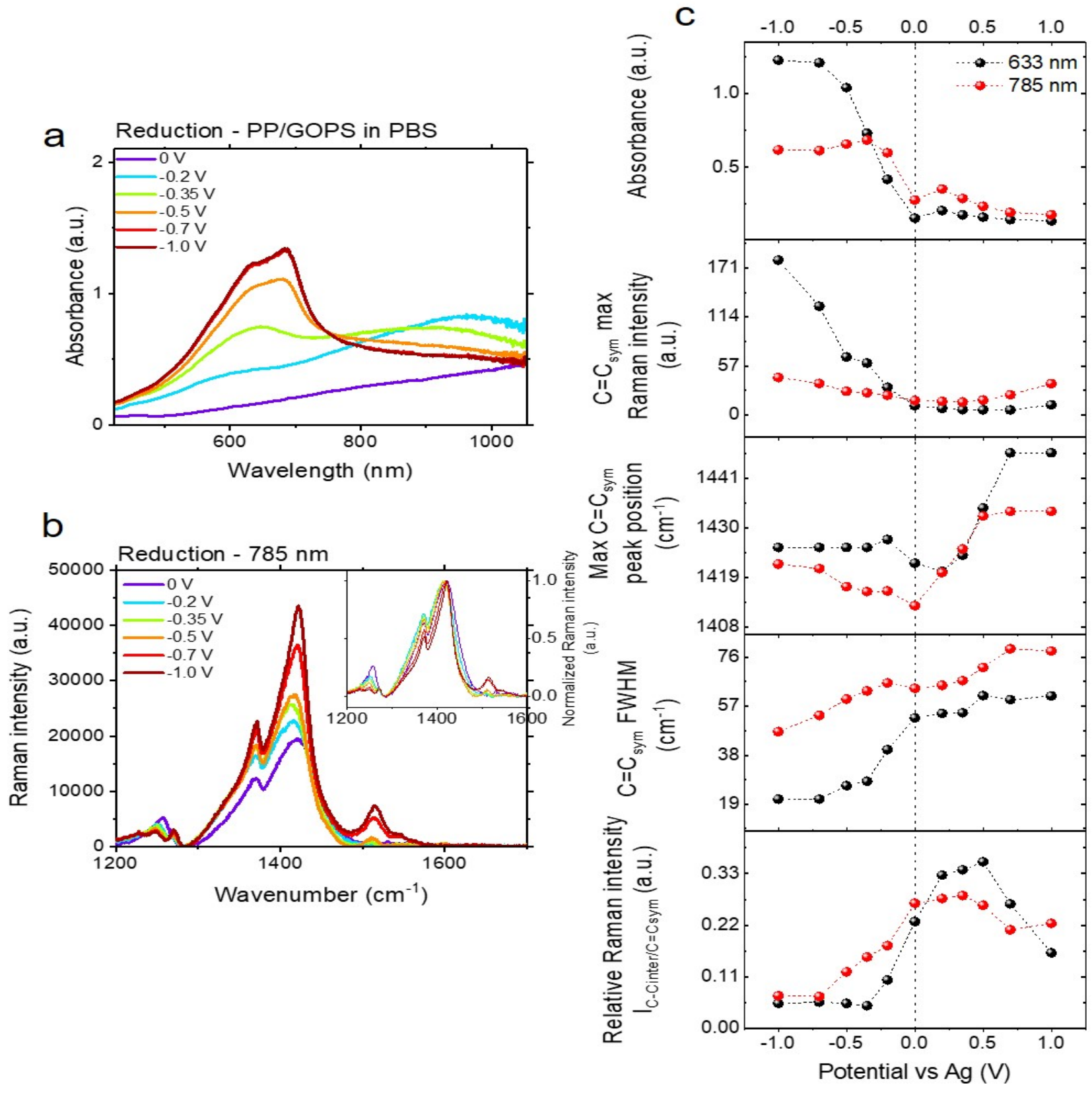

This article is protected by copyright. All rights reserved. 
Figure 2. a) An illustration of the 3 terminal OECT device configuration with an electrolyte encapsulated by the polydimethylsiloxane (PDMS) well, positioned under the Raman laser for in-situ measurements and the schematic of the ferrocene mediated mechanism of the glucose conversion within the well. b) Chronopotentiometric recording plotted on a log scale for the glucose sensing with a ferrocene mediated OECT sensor $\left(\mathrm{V}_{\mathrm{g}}=0.8 \mathrm{~V}\right.$ and $\left.\mathrm{V}_{\mathrm{SD}}=-0.3 \mathrm{~V}\right)$. The difference between the baseline after preconditions and the drain current after subsequent glucose addition represents the sensing regime (yellow). c) The in-situ Raman spectra during device operation at each stage of the experiment. d) A summary of the $C=\mathrm{C}_{\text {sym }}$ FWHM, relative peak areas of $\mathrm{C}=\mathrm{C}_{\text {low }}$ to $\mathrm{C}=\mathrm{C}_{\text {high }}$ and average drain current changes at each stage of the glucose sensing experiment with uncertainties from the extraction of Raman peak parameters and fluctuations in the drain current.
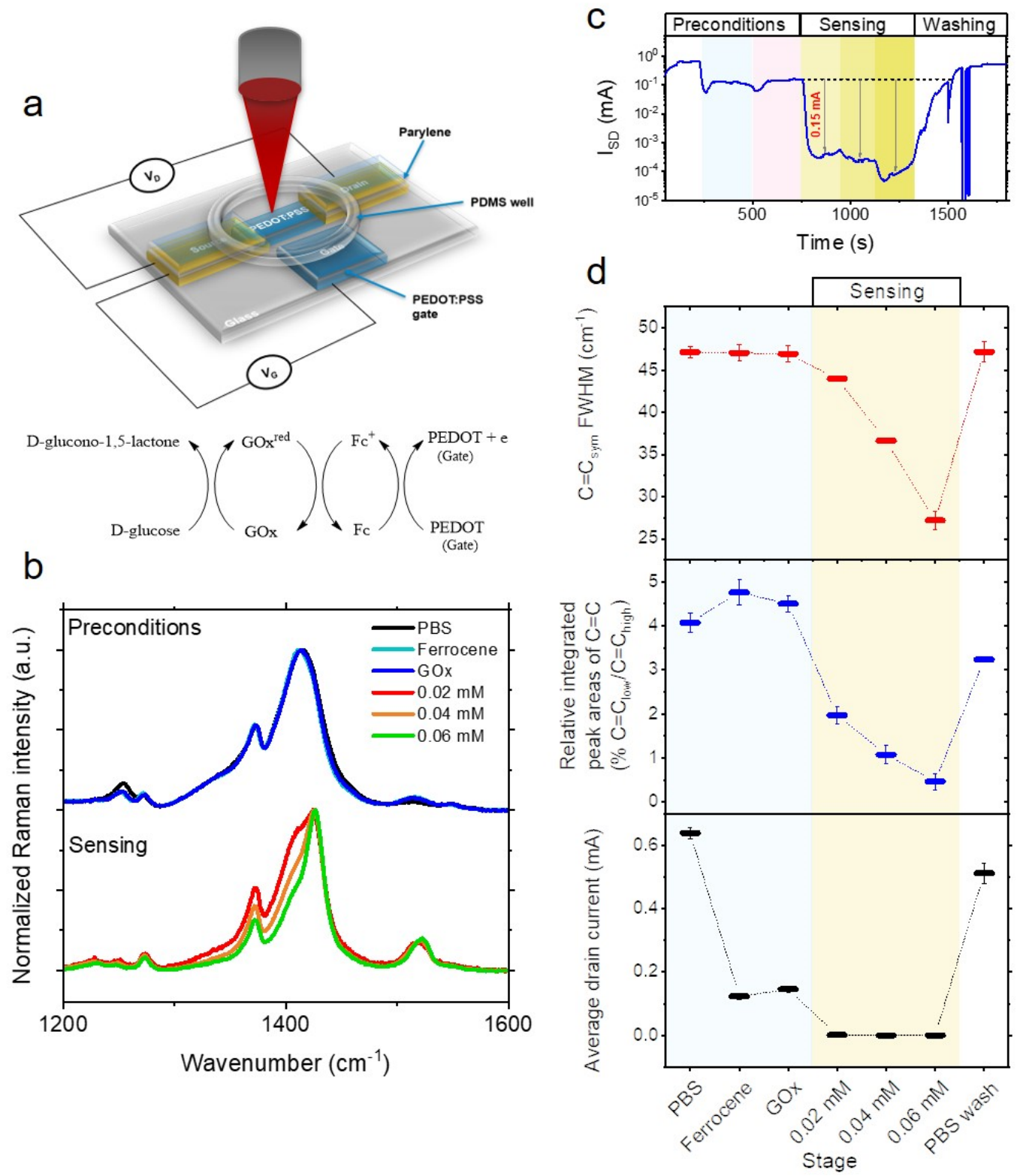

This article is protected by copyright. All rights reserved. 
Figure 3. a) Chronopotentiometric recording plotted on a log scale for the glucose sensing with a ferrocene mediated OECT sensor and a total concentration of $15 \mathrm{mM}$ glucose in electrolyte well. The complex cell media contains 10\% FBS, L-glutamine, phenol red and smaller percentages of other amino acids, vitamins and inorganic salts. b) The in-situ Raman spectra during device operation at each stage of the experiment. c) A summary of the $\mathrm{C}=\mathrm{C}_{\text {sym }}$ FWHM, relative peak areas of $\mathrm{C}=\mathrm{C}_{\text {low }}$ to $\mathrm{C}=\mathrm{C}_{\text {high }}$ and average drain current changes at each stage of the glucose sensing experiment with uncertainties from the extraction of Raman peak parameters and fluctuations in the drain current.

a
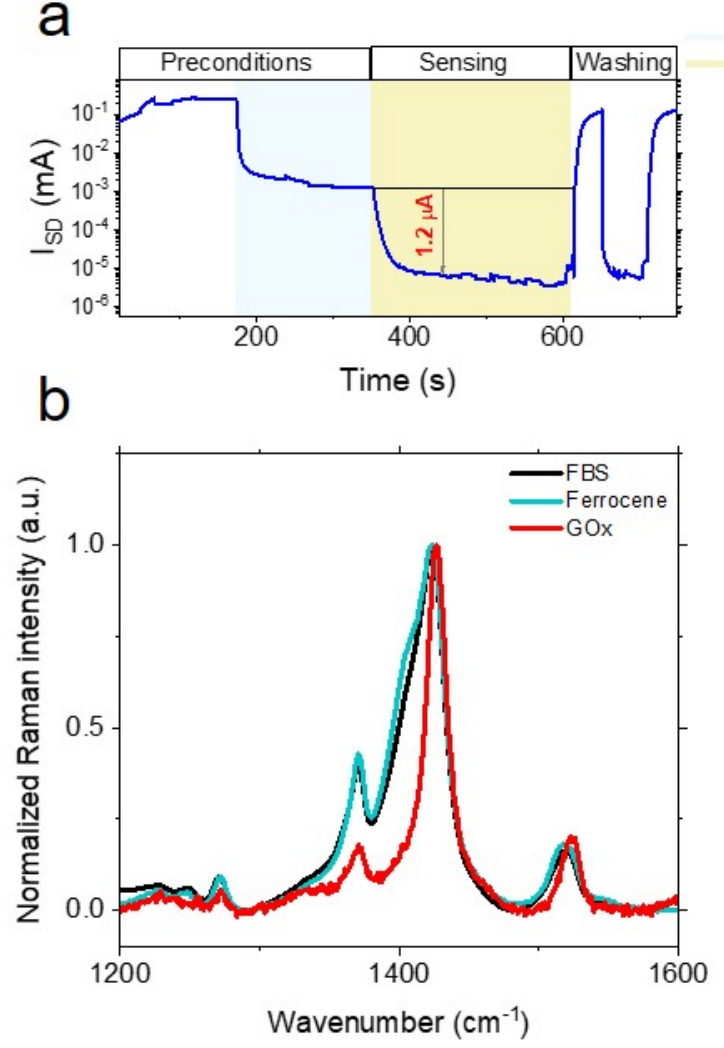

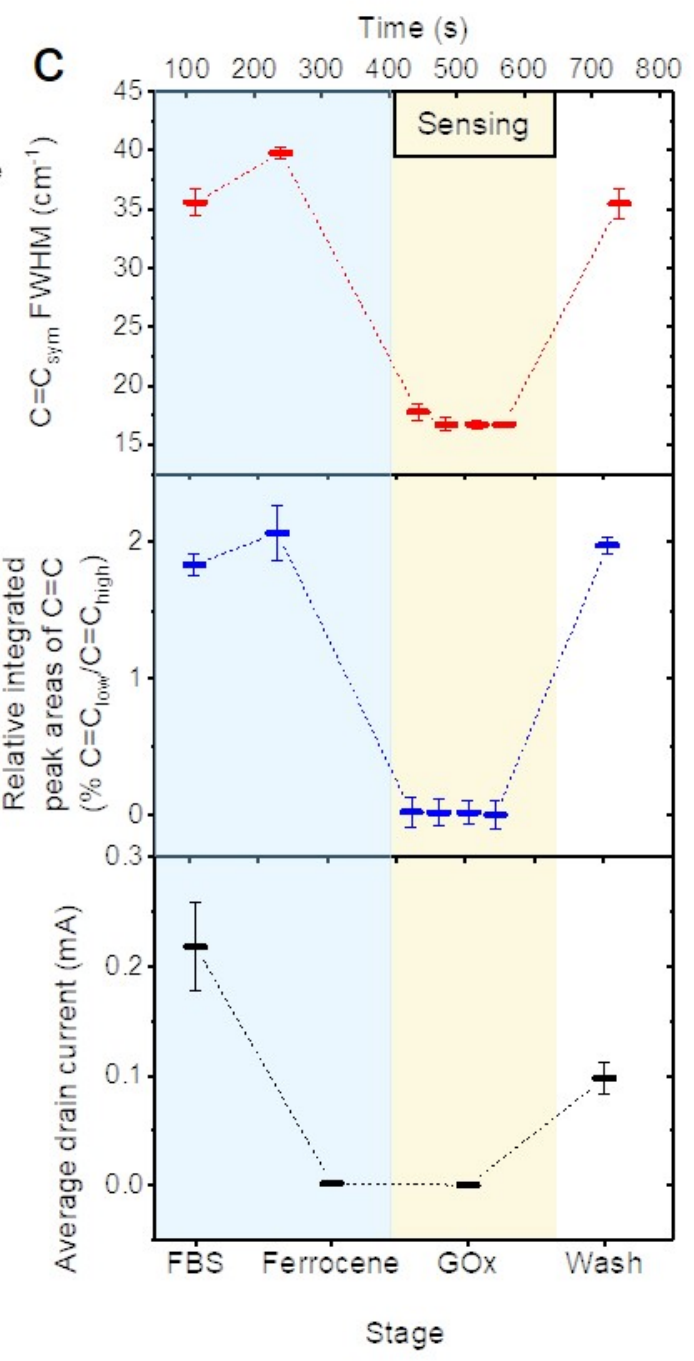

This article is protected by copyright. All rights reserved. 